Conclusion Despite personal experience with HIV, risky sexual behaviours and STI prevalence remain a problem in persons living with HIV in the capital city of Nicaragua. In order to prevent reinfection between serodiscordant partners as well as continued STI transmission, it is important for STI prevention programs to continue to develop their sexual health services, focusing on improved condom access and promotion.

\section{P1-S5.12 POPULATION ATTRIBUTABLE RISK FOR CHLAMYDIA INFECTION IN A COHORT OF YOUNG INTERNATIONAL TRAVELLERS (BACKPACKERS) AND RESIDENTS IN AUSTRALIA}

doi:10.1136/sextrans-2011-050108.190

H Wand. University of New South Wales, Sydney, Australia

Aim To estimate the population attributable risk (PAR) for Chlamydia trachomatis infection in young men and women in Sydney, Australia.

Method Multivariate logistic regression was used to examine the association between demographic, sexual behaviour and other potential risk factors on chlamydia positivity in young ( $\leq 30$ years) heterosexual international travellers (backpackers) and Australian residents attending a sexual health clinic. Point and interval estimates of PAR were calculated to quantify the proportion of chlamydia infections that can theoretically be prevented if a combination of risk factors is eliminated from a target population.

Results In males, the PAR associated with inconsistent condom use in the past 3 months was $65 \%$ (95\% CI 56 to 71\%) in backpackers compared to $50 \%$ (95\% CI 41 to $56 \%$ ) in non-backpackers and the PAR associated with reporting three or more female sexual partners in the past 3 months was similar between male backpackers and non-backpackers; 33\% (95\% CI 28 to 40\%) and 36\% (95\% CI 32 to $41 \%$ ), respectively. In females, the PAR associated with inconsistent condom use in the past 3 months was 51\% (95\% CI 42 to $59 \%$ ) in backpackers compared to $41 \%$ ( $95 \%$ CI 31 to $51 \%$ ) in non-backpackers, and the PAR associated with reporting three or more male sexual partners in the past 3 months was 14\% (95\% CI 11 to $18 \%$ ) in backpackers compared to $30 \%$ (95\% CI 25 to $37 \%$ ) in non-backpackers.

Conclusion These findings suggest that the largest number of chlamydia infections could be avoided by increasing condom use, particularly in backpackers. Reporting multiple partners was also associated with a large proportion of infections and the risk associated with this behaviour should be included in health promotion strategies.

\section{P1-S5.13 RECREATIONAL DRUG USE DURING SEX IS ASSOCIATED WITH STI AMONG CLIENTS OF A LARGE STI OUTPATIENT CLINIC IN AMSTERDAM, THE NETHERLANDS}

doi:10.1136/sextrans-2011-050108.191

\begin{abstract}
${ }^{1} \mathrm{M}$ Heiligenberg, ${ }^{1} \mathrm{P}$ Wermeling, ${ }^{1} \mathrm{M}$ van Rooijen, ${ }^{1} \mathrm{~A}$ Urbanus, ${ }^{1} \mathrm{M}$ Prins, ${ }^{2} \mathrm{R}$ Coutinho, ${ }^{1}$ M Schim van der Loeff. ${ }^{1}$ Public Health Service Amsterdam, Amsterdam, Netherlands; ${ }^{2}$ National Institute for Public Health and the Environment, Bilthoven, Netherlands
\end{abstract}

Background Previous studies have shown that recreational drug use is associated with high-risk sexual behaviour and STI. Little is known about the relationship between the use of specific recreational drugs during sex, sexual behaviour and the risk for any STI (chlamydia (CT), gonorrhoea (NG) and/or syphilis) or the three
STI separately in heterosexual men, men having sex with men (MSM) and women. We assessed the prevalence of recreational drug use during sex, and assocations of drug use during sex with high-risk sexual behaviour and STI.

Methods Attendees of the STI clinic of the Public Health Service of Amsterdam were interviewed during three waves of a bi-annual anonymous survey (2008-2009) about sexual behaviour in the preceding 6 months (number of steady and casual sex partners, condom use during vaginal or anal sex) and specific recreational drug use just before or during sex in the preceding 6 months (cannabis, XTC, poppers, heroine, cocaine, GHB, apomorfine, amphetamine, methylamphetamine, mushrooms, ketamine and explosion). Participants were tested for CT, NG and syphilis. Associations between drug use during sex and high-risk sexual behaviour and STI were analysed using multivariable logistic regression analyses. Analyses were done separately for heterosexual men, men who have sex with men (MSM) and women.

Results In total, 1012 heterosexual men, 749 MSM and 1254 women participated in this study. Of these, $11.7 \%$ had CT, 3.5\% NG and $1.4 \%$ syphilis. Recreational drug use during sex in the previous 6 months was reported by $22.5 \%$ of heterosexual men, $52.7 \%$ of MSM and $15.6 \%$ of women. In all three groups, drug use during sex was associated with unprotected vaginal and anal sex and more casual sex partners. In multivariable analyses among MSM, adjusting for age, ethnicity, educational level and sexual behaviour, poppers use was associated with any STI (adjOR 1.8, 95\% CI 1.2 to 2.6) and with NG (adjOR 2.6, 95\% CI 1.6 to 4.2), and GHB use with syphilis (adjOR 2.3, 95\% CI 1.0 to 5.0 ). In multivariable analyses among women, GHB use was associated with any STI (adjOR 4.7, 95\% CI 1.8 to 12.2 ) and CT (adjOR 3.5, 95\% CI 1.1 to 11.4). In heterosexual men, drug use during sex was not associated with STI.

Conclusions STI clinic clients frequently report recreational drug use during sex and this is associated with high-risk sexual behaviour in heterosexual men, MSM and women. The use of drugs during sex is associated with STI in MSM and women, but not in heterosexual men.

\section{P1-S5.14 RISK FACTORS ASSOCIATED WITH HIV ACOUISITION: A COMPARATIVE ANALYSIS OF OLDER AND YOUNGER WOMEN WHO PARTICIPATED IN THE MDP301 TRIAL IN JOHANNESBURG}

doi:10.1136/sextrans-2011-050108.192

${ }^{1} \mathrm{~S}$ Delany-Moretlwe, ${ }^{1} \mathrm{~A}$ Nanoo, ${ }^{2} \mathrm{~A}$ Nagpal, ${ }^{1} \mathrm{H}$ Moultrie, ${ }^{1} \mathrm{~S}$ Delany, ${ }^{1} \mathrm{H}$ Rees. ${ }^{1} \mathrm{WHI}$, Hillbrow, South Africa; ${ }^{2}$ LSHTM, UK

Background Young women are known to be particularly at risk for HIV infection. Identifying characteristics associated with HIV-acquisition in younger and older women could assist with developing effective interventions which target the determinants of HIV.

Methods The MDP301 study was an international, multi-centre, randomised controlled trial to assess the efficacy of $0.5 \%$ PRO2000/ 05 microbicide gel. 2508 HIV-negative women, $\geq 18$ years, were enrolled in Soweto and Orange Farm and followed up for 12 months. Associations between baseline demographic, behavioural and clinical risk factors and HIV acquisition were assessed using univariate Poisson regression.

Results Data on 2451 women were analysed. 110 seroconversions were observed over 2356.5 woman-years (wy). Overall, HIV incidence was $46.7 / 1000$ wy (95\% CI 38.7 to 56.3). Younger women (18-24 years) were more likely to acquire HIV (IRR1.4, 95\% CI 1.0 to 2.0) than older women ( $\geq 25$ years). Difficulty accessing money for medical treatment (IRR $1.5,95 \%$ CI 0.06 to $2.0, p=0.019$ ) 
and being HSV2 seropositive at baseline (IRR1.7, 95\% CI 0.99 to 2.8, $\mathrm{p}=0.055$ ) were associated with an increased risk of seroconversion in younger women. In older women, decreasing partner contribution to the household economy (IRR 1.3, 95\% CI 1 to 1.7) and Trichomonas vaginalis infection at enrolment (IRR 2.3, 95\% CI 1.1 to 4.9) were associated with increased risk of HIV acquisition. Older women who reported condom use at last sex act had a greater risk of acquiring HIV (IRR 2.8, 95\% CI 1.3 to 5.9), which could reflect high risk sexual behaviour in this group. Reporting multiple partners was associated with increased risk of $\mathrm{HIV}$ acquisition in both younger (2.6, 1.3 to 5.3 ) and older women (2.8, 1.3 to 6.1$)$.

Conclusions STIs remain an important marker of ongoing HIV risk. Interventions that address the economic context of women's HIV risk will be important to evaluate. Additional investigation using multivariate analysis may elucidate these findings further.

\section{P1-S5.15 CONTRIBUTION OF CONDOM BREAKAGE TO THE HIV EPIDEMIC AMING MEN WHO HAVE SEX WITH MEN IN KARNATAKA, INDIA}

doi:10.1136/sextrans-2011-050108.193

${ }^{1} \mathrm{M}$ Pickles, ${ }^{2} \mathrm{P}$ Vickerman, ${ }^{3} \mathrm{~J}$ Bradley, ${ }^{4} \mathrm{~B} M$ Ramesh, ${ }^{4} \mathrm{~S}$ Isac, ${ }^{5} \mathrm{R}$ Washington, ${ }^{2} \mathrm{H}$ Prudden, ${ }^{2} \mathrm{~K}$ Mitchell, ${ }^{6} \mathrm{M}$ Alary, ${ }^{1} \mathrm{M} \mathrm{C}$ Boily. ${ }^{1}$ Imperial College, London, UK; ${ }^{2}$ LSHTM, UK; ${ }^{3}$ CHARME-India project, Bangalore, India; ${ }^{4}$ Karnataka Health Promotion Trust, Bangalore, India; ${ }^{5}$ St. John's Medical College Bangalore, India; ${ }^{6}$ Centre Hospiltalier Affilié Universitaire de Québec, Quebec, Canada

Background Condom promotion among female sex workers (FSWs) and men who have sex with men (MSM) is one of the key components of the Avahan HIV intervention currently underway in India. High rates of consistent condom use are reported across sites. However, condoms are only effective if they do not break or slip during intercourse, which occur frequently, especially in MSM.

Methods We use a deterministic compartmental mathematical model of MSM divided into high and low risk, with average condom use increasing over time, to investigate how breakage influences the course of the epidemic. High-risk MSM (HRMSM) sell sex or have many casual partners, and typically have mostly receptive anal sex. Prior parameter ranges (including condom use) for the model are based on one round of cross-sectional behavioural and biological (IBBA) surveys of HRMSM from four districts in Karnataka state in 2008. In these surveys $16.7 \%$ (82/491) of MSM report condom breakage in the last sex act. Condom use increased to $93 \%$ by 2008 , and was assumed to be constant afterwards. The prior ranges are sampled using Latin hypercube sampling. Model runs that agree with measured HRMSM HIV prevalence (12.7-18.9\%) provide the posterior parameter set fitting the data, and are used to make predictions of HIV prevalence with and without condom breakage after the start of the intervention in 2004.

Results Abstract P1-S5.15 figure 1 shows change in high/low risk prevalences with and without condom breakage. Even with high rates of condom breakage, HIV prevalence among MSM declines from $16.7 \%$ (95\% CI 12.7 to $20.8 \%$ ) in 2004 to $7.9 \%$ (5.9 to $9.8 \%$ ) in HRMSM by 2015. Without condom breakage HIV prevalence declines faster, to $5.6 \%$ (4.2 to $6.9 \%$ ) by 2015 . Compared to no condom breakage, there will be $10.3 \%(10.7 \%)$ more cumulative infections with breakage in high-risk (low-risk) MSM from 2004 until 2015. The annual extra fraction of new infections due to condom breakage increases from $4.1 \%$ (4.3\%), in 2004, to $158.9 \%$ (165.3\%), in 2015 in high-risk (low-risk) MSM, although the absolute number of infections due to condom breakage decreases as the epidemic is declining and condom use increases.

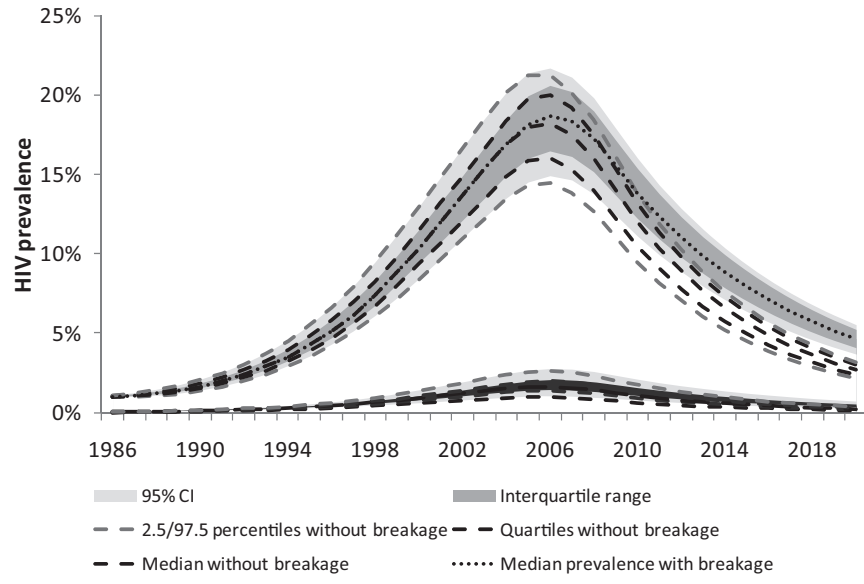

Abstract P1-S5.15 Figure 1 HIV prevalence against time for high-risk MSM (upper curves)and low-risk MSM (lower curves). Shaded region shows 2.5-97.5 percentiles and IQR with breakage. The dashed lines show the 2.5, 97.5 percentiles and quartiles without condom breakage.

Conclusions HIV prevalence is projected to decline in MSM in Karnataka, given high rates of reported condom use, even with frequent breakage. However, there will be a large fraction of extra infections due to condom breakage. HIV intervention programmes should examine reasons for high rates of breakage and take steps to address this.

\section{P1-S5.16 INVESTIGATING SELF-REPORTED LEVEL OF CONDOM USE AND CONDOM USE IN LAST ACT AMONG HIGH-RISK GROUPS IN SOUTHERN INDIA}

doi:10.1136/sextrans-2011-050108.194

${ }^{1} \mathrm{M}$ Pickles, ${ }^{2} \mathrm{R}$ Adhikary, ${ }^{3} \mathrm{M}$ Mainkar, ${ }^{3} \mathrm{R} S \mathrm{P},{ }^{4} \mathrm{~K}$ Deering, ${ }^{5} \mathrm{~S}$ Verma, ${ }^{1} \mathrm{M} \mathrm{C}$ Boily, ${ }^{6} \mathrm{P}$ Vickerman, ${ }^{7} \mathrm{M}$ Alary. ${ }^{1}$ Imperial College, London, UK; ${ }^{2}$ Family Health International, India; ${ }^{3}$ National AIDS Research Institute, Pune, India; ${ }^{4}$ University of British Columbia, Canada; ${ }^{5}$ Karnataka Health Promotion Trust, Bangalore, India; ${ }^{6}$ LSHTM, UK; ${ }^{7}$ Centre Hospiltalier Affilié Universitaire de Québec, Quebec, Canada

Background Measuring consistent condom use (CCU) is crucial in understanding the trajectory of the HIV epidemic and in evaluating HIV preventive interventions that promote condom use. We examine how one measure of CCU varies across different populations, and compare it to condom use in the last sex act.

Methods Using data from cross-sectional behavioural and biological surveys (2005-2007), we compare responses concerning selfreported level of condom use and condom use in the last act by female sex workers (FSWs), their clients, and men who have sex with men (MSM) in districts from Andhra Pradesh, Maharashtra and Tamil Nadu states in India. For FSWs/clients, the question related to sex with occasional clients/FSWs. For MSM, the question was for non-commercial, non-regular male partners. The time period was unspecified. Levels of condom use were defined as every time", "most of the time/sometimes", and "never". We define CCU as those who reply "every time".

Results $95.2 \%$ of all FSWs reported using a condom in the last act, compared to $72.9 \%$ of clients and $80.0 \%$ MSM. However, only $36.7 \%$ of clients and $29.6 \%$ of MSM reported CCU compared to $80.0 \%$ of FSWs. Abstract P1-S5-16 table 1 shows condom use in the last act stratified by reported level of condom use. For all populations reporting CCU "always", condom use in the last act was $>98 \%$. Of those who reported "most of the time/sometimes", there was a significant difference between condom use in the last act between clients and the other groups ( $\chi^{2}$ test, 